\title{
THE LACK OF SELF-ADJOINTNESS IN THREE POINT BOUNDARY VALUE PROBLEMS
}

\section{ANTON ZETTL ${ }^{1}$}

In a paper with the same title, to appear shortly in the Pacific Journal of Mathematics, J. W. Neuberger showed that the problem:

$$
\begin{aligned}
\left(p y^{\prime}\right)-q y & =g \text { with } A\left[\begin{array}{c}
(y a) \\
p(a) y^{\prime}(a)
\end{array}\right]+B\left[\begin{array}{c}
y(b) \\
p(b) y^{\prime}(b)
\end{array}\right]+C\left[\begin{array}{c}
y(c) \\
p(c) y^{\prime}(c)
\end{array}\right] \\
& =\left[\begin{array}{c}
0 \\
0
\end{array}\right] \text { where } a<b<c, p(t) \neq 0
\end{aligned}
$$

for $t$ in $[a, c], p, q, g$ are real continuous functions on $[a, c]$ and $A, B$, $C$ are real $2 \times 2$ matrices; in the determinate case, has a nonsymmetric Green's function if $B \neq 0$. The purpose of this paper is to generalize his result to higher order problems.

Consider problems:

( $\left.^{*}\right) Y^{\prime}=F Y$ with $A_{1} Y(a)+B_{1} Y(b)+C_{1} Y(c)=0$ where $A_{1}, B_{1}, C_{1}$ and $k \times k$ matrices, $k>1, Y$ is a $k$-dimensional vector and $F=\left(f_{i j}\right)$ satisfies

$$
\begin{aligned}
& f_{i j} \in C_{[a, c]}, f_{i j}=0 \text { if } j>i+1 \text { or } i+j \text { is even, } \\
& f_{i, i+1}(t) \neq 0 \text { for } t \in[a, c], \quad i, j=1, \cdots, k \text { and }
\end{aligned}
$$

(**) $X^{\prime}=H X$ with $A_{2}(X)(a)+B_{2} X(b)+C_{2} X(c)=0$ where $H=\left(f^{*}{ }_{k+1-j, k+1-i}\right), A_{2}, B_{2}, C_{2}$ are $k \times k$ matrices. Observe that $H$ also satisfies (1).

In [1] the author has shown that every equation of the form $L y=\sum_{i=1}^{k} p_{i} y^{(i)}=0$ with $p_{i} \in C_{[a, c]}, p_{k}(t) \neq 0, t \in[a, c]$, has a vectormatrix representation of the type $Y^{\prime}=F Y$ with $F$ satisfying (1), and that $X^{\prime}=H X$ serves as an "adjoint" equation.

Let $M=\left(m_{i j}\right)$ and $N=\left(n_{i j}\right)$ denote the unique matrix functions (see [31]) such that $M(t, u)=I+\int_{u}^{t} F(s) M(s, u) d s$ and $N(t, u)$ $=I+\int_{u}^{t} H(s) N(s, u) d s$ for all $t, u$ in $[a, c]$. Throughout this paper we will assume that $A=\left[A_{1}+B_{1} M(b, a)+C_{1} M(c, a)\right]^{-1}$ and $B$ $=\left[A_{2}+B_{2} N(b, a)+C_{2} N(c, a)\right]^{-1}$ exist. The existence of these inverses is equivalent to problems $\left({ }^{*}\right)$ and $\left({ }^{* *}\right)$, respectively, having no nontrivial solutions. (The proof of this fact is entirely analogous to the 2-point case, see [4], and is therefore omitted.)

Let $V=-A C_{1} M(c, a), W=-A B_{1} M(b, a), U=-B C_{2} N(c, a)$, $Z=-B B_{2} N(b, a)$.

Presented to the Society, January 25, 1966; received by the editors October 4, 1965.

${ }^{1}$ Research for this paper was supported in part by NASA grant NGR-19-001-011. 
Define
(2) $L(t, x)= \begin{cases}M(t, a) V M(a, x) & b<x \leqq c, a \leqq t \leqq c ， \\ M(t, a)(V+W) M(a, x) & a \leqq x \leqq b, a \leqq t \leqq c ;\end{cases}$
(3) $J(t, x)= \begin{cases}N(t, a) U N(a, x) & b<x \leqq c, a \leqq t \leqq c ， \\ N(t, a)(U+Z) N(a, x) & a \leqq x \leqq b, a \leqq t \leqq c ;\end{cases}$
(4) $\quad K(t, x)= \begin{cases}L(t, x) & a \leqq t<x \leqq c ; \\ L(t, x)+M(t, x) & a \leqq x \leqq t \leqq c ;\end{cases}$
(5) $G(t, x)= \begin{cases}J(t, x) & a \leqq t<x \leqq c, \\ J(t, x)+N(t, x) & a \leqq x \leqq t \leqq c .\end{cases}$

We remark that $K$ is a kernel function for $\left(^{*}\right)$ i.e. for any continuous vector $G$ the solution $Y=\left(y_{i}\right)$ of $Y^{\prime}=F Y+G$ with $A_{1} Y(a)+B_{1} Y(b)$ $+C_{1} Y(c)=0$ is given by $Y(t)=\int_{a}^{c} K(t, s) G(s) d s$ for $t$ in $[a, c]$. The proof, again, is analogous to the 2-point case given in [4]. Observe that for

$$
G=\left[\begin{array}{c}
0 \\
0 \\
\vdots \\
0 \\
g
\end{array}\right], \quad y_{1}(t)=\int_{a}^{c} K_{1 k}(t, s) g(s) d s,
$$

so that $K_{1 k}$ is the Green's function of an ordinary boundary value problem.

TheOREM 1. If either $B_{1} \neq 0$ or $B_{2} \neq 0$ then there exist $t, x$ in $[a, c]$ such that

$$
K_{1 k}(t, x) \neq(-1)^{k}\left(G_{1 k}^{*}(x, t)\right) .
$$

The following results are used in the proof of Theorem 1.

$$
N(u, t) N(t, x)=N(u, x) \text { for any } t, u, x \in[a, c]
$$

(see [3]).

(7) $m_{i j}(t, x)=(-1)^{i+j} n_{k+1-j, k+1-i}^{*}(x, t)$ for $x, t$ in $[a, c], i, j=1, \cdots, k$. (See [2], p. 12.)

(8) For a given $u \in[a, c], m_{1 i}(t, u), i=1, \cdots, k$ are linearly independent on any subinterval of $[a, c]$.

Proof of Theorem 1. Suppose $K_{1 k}(t, x)=(-1)^{k} G_{1 k}^{*}(x, t)$ for all 
$t, x$ in $[a, c]$. Then, using (2), (3), and (7) $L_{1 k}(t, x)=(-1)^{k}\left[J_{1 k}^{*}(x, t)\right.$ $\left.+n_{1 k}^{*}(x, t)\right] x, t \in[a, c]$. For $a \leqq x \leqq b, b \leqq t \leqq c$, using (2), (3), (6), and (7), we have

$$
\begin{aligned}
\sum_{i=1}^{k} m_{1 i}(t, a) & \sum_{j=1}^{k}\left(v_{i j}+w_{i j}\right) m_{j k}(a, x) \\
= & (-1)^{k} \sum_{i=1}^{k}(-1)^{1+i} m_{1 i}(t, a) \sum_{=1}^{k}(-1)^{j+k} m_{j, k}(a, x) u_{k+1-j, k+1-i}^{*} \\
& -\sum_{i=1}^{k} m_{1 i}(t, a) m_{i k}(a, x) .
\end{aligned}
$$

Hence, using $8, w_{i j}=(-1)^{i+j+1} u_{k+1-j, k+1-i}^{*}-\delta_{i j}-v_{i j}$. Similarly for $a<x \leqq c$ and $b<t \leqq c$ we get $v_{i j}=(-1)^{i+i+1} u_{k+1-j, k+1-i}^{*}-\delta_{i j}$. Hence $W=0$ and $B_{1}=0$.

Similar considerations lead to $B_{2}=0$. This contradiction completes the proof.

Theorem 1 can be generalized as follows: Instead of $\left(^{*}\right)$ and $(* *)$ consider

I. $Y^{\prime}=F Y$ with $\sum_{i=1}^{m} A_{i} Y\left(a_{i}\right)=0$ where $A_{i}, i=1, \cdots, m, m>2$, are $k \times k$ matrices and $a=a_{1}<a_{2}<\cdots<a_{m}=c$ and

II. $X^{\prime}=H X$ with $\sum_{i=1}^{m} B_{i} X\left(a_{i}\right)=0 ; B_{i}, i=1, \cdots, m$ are $k \times k$ matrices. Construct kernel functions $K$ and $G$, for Problems I and II, respectively, analogously to (4) and (5). We have

Theorem 2. If at least one of $A_{i}, B_{i}, i=2, \cdots, m-1$ is not zero, then there exist $t, u \in[a, c]$ such that $K_{1 k}(t, u) \neq(-1)^{k}\left(G_{1 k}^{*}(u, t)\right)$.

The proof is similar and is therefore omitted.

In the rest of the paper we develop similar results for the Green's matrices $K$ and $G$ as a whole-rather than just the upper right hand corner $K_{1 k}$ and $G_{1 k}$.

Henceforth $F=(f i j)$ is any $k \times k$ matrix of continuous complex valued functions. Let $H=\left((-1)^{i+i} \bar{f}_{k+1-j, k+1-i}\right) ; M, N, K$ and $G$ are defined as above. Let $T=\left((-1)^{i} \delta_{i, k+1-j}\right)$.

THEOREM 3. If either $B_{1} \neq 0$ or $B_{2} \neq 0$, then there exist $t, x$ in $[a, c]$ such that

$$
K(t, x) \neq-T^{-1} G^{*}(x, t) T .
$$

Note. In the proof of Theorem 3 we use the fact that $M(t, x)$ $=T^{-1} N^{*}(x, t) T$-a restatement of (7). This is proven in $[2, \mathrm{p} .12]$ only under a more restrictive condition on $F$. However, the extension of 
the proof to the present setting is straightforward-the pertinent fact being that $H=-T^{-1} F^{*} T$.

Proof of Theorem 3. Suppose $K(t, x)=-T^{-1} G^{*}(x, t) T$ for every $t, x$ in $[a, c]$.

For $a<x<b$ and $b<t<c$ we have

$$
\begin{aligned}
K(t, x) & =L(t, x)+M(t, x)=M(t, a)(V+W) M(a, x)+M(t, x) \\
& =M(t, a)[V+W+I] M(a, x), \\
G(x, t) & =N(x, a) U N(a, t) .
\end{aligned}
$$

A simple computation, see (7) above, yields

$$
-T^{-1} G^{*}(x, t) T=M(t, a)\left[-T^{-1} U^{*} T\right] M(a, x) .
$$

Hence $V+W+I=-T^{-1} U^{*} T$.

On the other hand for $b<t<c, b<x<c$, we have

$$
\begin{aligned}
& K(t, x)=L(t, x)=M(t, a) V M(a, x), \\
& G(x, t)=J(x, t)+N(x, t)=N(x, a) U N(a, t)+N(x, t) .
\end{aligned}
$$

A similar computation yields:

$$
-T^{-1} G^{*}(x, t) T=M(t, a)\left[-T^{-1} U^{*} T-I\right] M(a, x) .
$$

Hence $V=-T^{-1} U^{*} T-I$; and therefore $W=0=B_{1}$. Similarly one can show that $B_{2}=0$. This contradiction completes the proof.

Theorem 3 readily extends to $n$ point problems for any positive integer $n>2$.

\section{REFERENCES}

1. J. W. Neuberger, Concerning boundary value problems, Pacific J. Math. 10 (1960), 1385-1392.

2. H. S. Wall, Concerning harmonic matrices, Arch. Math. 5 (1954), 160-167.

3. Anton Zettl, Adjoint linear differential operators, Proc. Amer. Math. Soc. 16 (1965), 1238-1240.

4. - - Related two-point boundary value problems and harmonic matrices, Doctoral Dissertation, University of Tennessee, Knoxville, 1964.

Louisiana State University 